\title{
Die Vagus-Meditation
}

\section{Eine Chance gegen Stress und Burnout im Klinikalltag}

Laut Gesundheitsreport der BKK von 2011 stehen die Fehlzeiten durch Erkrankungen des Stütz- und Bewegungsapparates mit 26,6\% nach wie vor an der Spitze, die psychosomatischen Erkrankungen liegen bei $12 \%$. Besorgniserregend ist jedoch deren Anstieg in den letzten 30 Jahren um das 3-Fache. Auffällig ist dabei der Anstieg von Depressionen, Schlafstörungen, Angststörungen und Burnout durch die ständige Arbeitsverdichtung mit gleichzeitiger Sorge um den Arbeitsplatz.
Neben Bewegung, Ernährung und Sozialkontakten mit anderen Menschen ist es vor allem die Meditation, die als „Zündschlüssel“ für ein positives Lebensgefühl wirkt. Inzwischen hat auch die westliche Medizin den Stellenwert der Meditation besonders in der Prävention stressbedingter Erkrankungen erkannt. Studien auf diesem Gebiet nehmen weltweit zu. Einig ist man sich inzwischen darüber, dass durch Meditation eine hohe Schutzwirkung gegen Stress und Burnout erreicht werden kann. Ferner hat man erkannt, dass der Vagusnerv die Führungsrolle bei diesen Krankheiten einnimmt - nur kennt man noch nicht den Mechanismus, wie man diesen 10. Hirnnerven willentlich in Szene setzen kann. Eine Möglichkeit dazu kann die Vagus-Meditation sein, die den neuro-physiologischen Kanal zum Kerngebiet des Vagus im Hirnstamm belegt.

\section{N. vagus als Führungsnerv im} parasympathischen System

Der N. vagus ist der Führungsnerv im parasympathischen System, in ihm verlaufen $75 \%$ aller parasympathischen Fasern. Aus gutem Grund bezeichnet man ihn auch als den „Vagabunden“ unter den
Nerven, weil er sich im ganzen Körper „umhertreibt“. Zum anderen sind bestimmte Nerven im parasympathischen System nicht eigenständig, sie benutzen vielmehr andere motorische Nerven als „Dienstleister“, d.h. parallel zu motorischen Fasern von 3 Hirnnerven erreichen parasympathische Nerven das Kerngebiet im Hirnstamm (Abb. 1).

\section{Hirnnerv}

Im 3. Hirnnerven, dem motorischen Augennerv (N. oculomotorius), erreichen parasympathische und motorische Fasern die Okulomotoriuskerne, den parasympathischen Edinger-Westphal-Kern sowie den motorischen Okulomotoriuskern im oberen Pol des Hirnstamms. Die parasympathischen Fasern werden hinter dem Auge im Ganglion ciliare gebündelt, von hier aus erreichen sie den Ciliarmuskel, der die Augenlinse steuert, den Pupillenschließmuskel und den medialen, äußeren Augenmuskel, der den Augapfel zur Nahakkommodation nach innen dreht. Dieser Konvergenzvorgang ist gekoppelt mit der Anspannung des Ciliarmuskels, der die Linse auskugelt und mit der Aktivität des Pupillenschließmuskels kombiniert. Gleichzeitig überschneiden sich die Blickachsen, hervorgerufen durch die Verkürzung des medialen, äußeren $\mathrm{Au}-$ genmuskels, der ebenfalls parasympathische Fasern aufweist. Dieses Muskeltrio ist durch seine beigelegten parasympathischen Fasern imstande, im Falle der Aktivierung sofort eine Tiefenentspannung auszulösen.
Abb. 1 Verlauf der Hirnnerven zum Hirnstamm.
7. Hirnnerv

Auch der 7. Hirnnerv, der N. facialis, führt parasympathische Fasern, er versorgt die obere mimische Muskulatur um die Augen herum. Das „Lachen mit den Augen“ wird vom 7. Hirnnerven bestimmt, speziell beeinflusst von der seitlichen Schläfenmuskulatur, die verantwortlich ist für die Lachfalten am äußeren Augenrand.

\section{Hirnnerv}

Der 9. Hirnnerv, der N. glossopharyngeus, steuert motorisch und parasympathisch die Zunge und den Kehlkopf. Schnell entfaltet sich die Entspannung durch Zungenstretching, wenn die Zunge nach oben eingerollt und nach vorn gegen den oberen Gaumen gedrückt wird. Kehlkopfvibrationen sind besonders tiefenentspannend, denn neben den parasympathischen Fasern im 9. Hirnnerven wird der Kehlkopf auch direkt vom Vagus über dessen Kehlkopfast (Ramus pharyngesus) erreicht. Das ist die Anatomie der VagusMeditation (Abb. 1).

\section{Stimulation der 3 Hirnnerven löst Entspannung aus}

Auf diesen Kanälen kann eine komplexe Entspannung ausgelöst werden, komplex deshalb, weil neben Herz und Lunge auch praktisch der gesamte Bauchraum erreicht wird. Die Anlaufstation im Hirnstamm sind die Hirnnervenkerne. Der Informationsaustausch zwischen Edinger-Westphal-Kern im oberen Pol des Hirnstamms 


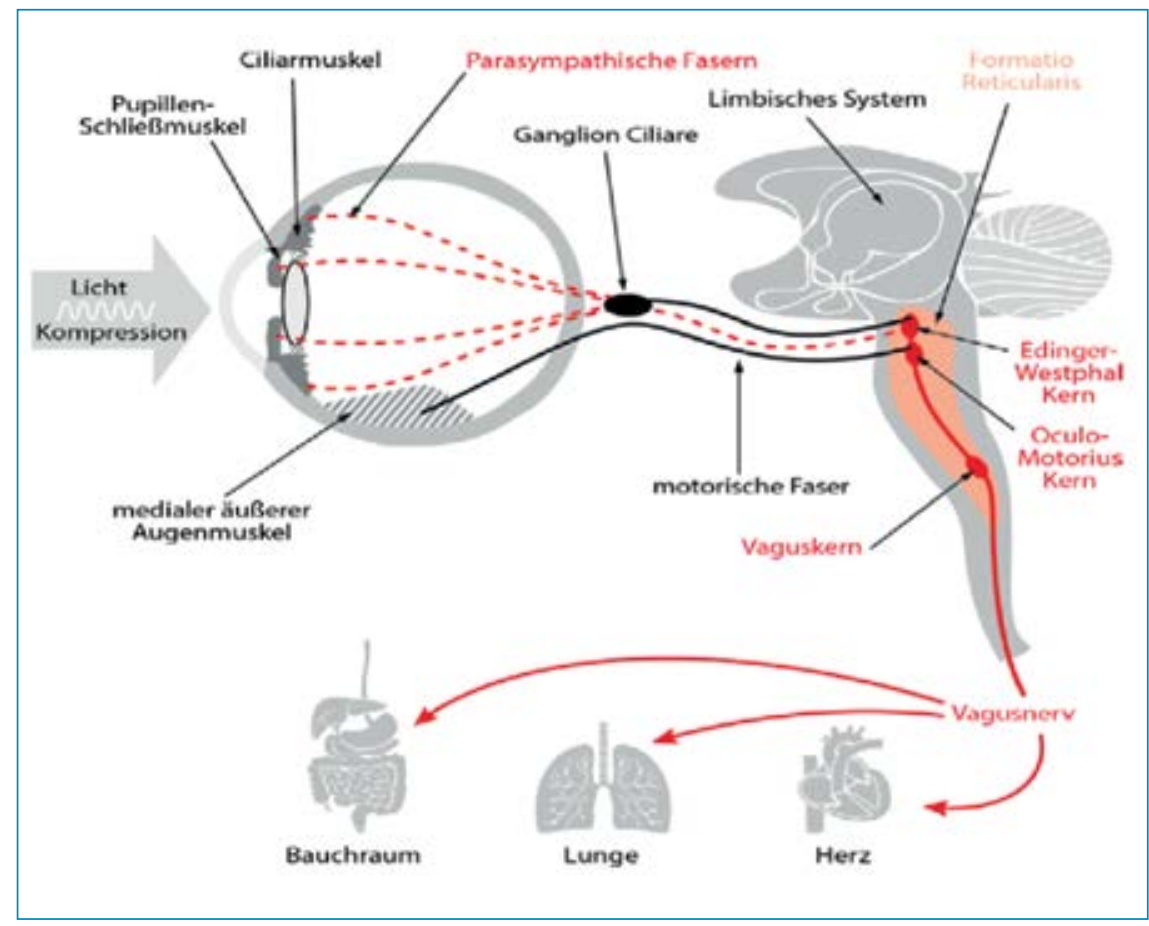

Abb. 2 Weiterleitung von Informationen durch den N. vagus an Herz, Lunge und Bauchraum.

und Vaguskern (Nucleus dorsalis N. vagi) erfolgt auf ganz bestimmten Bahnen, einmal gebildet von der Formatio reticularis sowie vom hinteren und mittleren Längsbündel (Fasziculus longitudinalis posterior et medialis). Der Vagus ist es dann, der die Information unmittelbar an Herz, Lunge und Bauchraum bis zur linken Dickdarmflexur weiterleitet (Abb. 2) und das mit folgendem Ergebnis:

- Beruhigung des Herzkreislaufsystems mit Blutdruckabfall und Absenkung der Herzfrequenz.

- Verlagerung der seitlichen Brustatmung in die tiefe Bauchatmung unter Betonung des Zwerchfells.

- Beschleunigung der Magen-Darmpassage.

- Verstärkung aller regenerativer Prozesse mit Muskelentspannung.

- Verbesserung der Kreativität durch Verlangsamung der Gehirnwellen. Das Tagesbewusstsein mit hoher Erregung des Betazustandes (15-30 Herz) wechselt in das beruhigende Alphastadium (7-15 Herz).

- Öffnung des Gedächtnisspeichers, der unter Stress schwer zugänglich ist.

- Rückbildung von chronischen Schmerzen.

\section{Techniken zur Anregung des Vagus im Hirnstamm} Die Stimulation der 3 Hirnnerven zur willentlichen Anregung des Vagus im Hirn- stamm kann durch folgende Techniken erreicht werden: Einmal durch die Konzentration auf die Atmung, zum anderen durch Kehlkopfvibrationen im Zusammenhang mit Mantras, die durch wiederholtes Murmeln oder Summen praktiziert werden.

Rein empirisch hat man in allen Religionen diese Möglichkeit komplexer Entspannung erkannt, ohne jedoch zu wissen, dass man sich stets auf den Bahnen des Vagus bewegte. Neu an diesen Techniken ist die schnelle und direkte Umsetzung im Stressalltag, die mit der klassischen Meditation praktisch kaum erreicht werden kann.

\section{"Cinema interne"} Bei geschlossenen Augen extreme Naheinstellung durch Fixierung der Augenlider von hinten. Zunächst ist alles grau in grau, bald tauchen
Farben auf, nicht selten das ganze Sonnenspektrum, das besonders bei Lichteinfall vor einem sonnendurchfluteten Fenster oder vor einer 100 Watt-Lampe in Erscheinung tritt. Auch Einschlüsse im Augenwasser, die „fliegenden Mücken“, können ins Visier genommen werden.

\section{Doppelbilder}

Doppelbilder prägen das Akkommodationstraining, wobei bald jeder Gegenstand an einer Wand akkommodiert werden kann. Verstärkend wirkt eine Achtertour der Augen um die beiden Gegenstände herum.

\section{Augenpressur}

Augenpressur erreicht seine unmittelbare Tiefenentspannung durch den zarten Druck beider Hände gegen die geschlossenen Augen, der mechanische Reiz trifft unmittelbar das Ganglion ciliare, in dem parasympathische Fasern gebündelt werden.

\section{Kehlkopfvibrationen}

Parallel zum „Cinema interne“ können auch Kehlkopfvibrationen den 9. Hirnnerven reizen (Abb. 3). Gefragt sind die 3 stimmungsvollen „S“, ausgelöst durch Schnurren, Summen, Singen. Hiermit verbunden ist eine tiefe Ausatmung, die vom Parasympathikus bestimmt ist, denn bei der nächsten Einatmung ist bereits wie- 


\section{Tab. $1 \quad$ Einsatz der Vagus-Meditation.}

- Am Arbeitsplatz in der Mittagspause zum Ausgleich des biologischen Tiefs, damit man in der zweiten Tageshälfte noch einmal durchstarten kann. 15 Minuten tiefe Entspannung, die die Vagus-Meditation sofort vermittelt, wirken stimmungsaufhellend und leistungssteigernd zugleich. Nach neuen Studien beträgt die Leistungssteigerung 35\%, die Gesundheitsförderung im Herz-Kreislaufsektor liegt bei $37 \%$.

- Bei Schlafstörungen aller Art.

- Gegen Sorgen und Ängste, wie Lampenfieber vor der Prüfung, Platzängste im der Röntgenröhre oder beim Zahnarzt.

- Flugangst während Start und Landung, Seekrankheit.

- Gegen die „Ungeduld-des-nicht-warten-Könnens“.

- Gegen die Lenkradmüdigkeit auf der Autobahn.

- Im Erkrankungsfall, denn jeder stationäre Aufenthalt ist mit langem Warten verbunden.

- Bei chronischen Schmerzen.

- Zur schnellen Regeneration nach einer Erkrankung oder nach großen Belastungen im Sport.

der der Sympathikus aktiv, so schnell schaltet das vegetative Nervensystem um. Neben dem Schnurren können auch kurze Melodien leise oder summend in ständiger Wiederholung genutzt werden. Gefragt sind die Klinger unserer Sprache, die Vokale A, O, U, dagegen ist das I in seiner Kopflage ein Stresston. Die Klinger versetzen den Brustraum in Schwingung, verstärkend wirken Imaginationen im Sinne des „erinnerten Wohlbefindens“, wenn Botschaften aus der Vergangenheit abgerufen werden, in denen man besonders glücklich war, denn das Gehirn kann nicht unterscheiden zwischen Vorstellungskraft und Realität.

\section{Zungenstretching}

Eine weitere Stimulation des 9. Hirnnerven erfolgt durch Zungenstretching. Hierbei wird die Zunge maximal nach vorn verlagert, eingerollt und gegen den oberen Gaumen gedrückt - eine ergänzende Episode, die jederzeit parallel zum Grundprogramm geschaltet werden kann.

\section{Anspannung der Schläfenmuskeln}

Eine unterstützende Episode ist die Anspannung der Schläfenmuskeln zur Aktivierung des 7. Hirnnerven (N. facialis), hierbei bewegt sich die Kopfhaut, die Ohren einbezogen. Gleichzeitig verschieben sich die Augenbrauen nach oben, sodass die stressbedingte Zornesfalte, die nicht selten Spannungskopfschmerzen verursacht, ausgeglichen werden kann.

\section{Wann ist die Vagus-Meditation angezeigt?}

Die Vagus-Meditation bietet eine Möglichkeit zur Regeneration. Sie wirkt schnell und komplex, sodass auch belas- tende Nachtdienste bei fortgesetzter Tagesarbeit überbrückt werden können (Tab. 1).

Natürlich braucht es eine gewisse Eingewöhnungszeit, um die Tiefenwirkung der Vagus-Meditation erfahren und schätzen zu können. Teilnehmer unserer Kurse belegen, dass sie bald nach Beginn der Übungen im Stressalltag in den gewünschten beruhigenden Erregungszustand kommen konnten, in dem sie alles um sich herum vergessen konnten. Dieser Bewusstseinszustand war dann nur noch von dem einen Gedanken beherrscht, dass dieser Moment nicht so schnell enden möge. Bei täglicher Praxis genügen dann nur noch wenige Minuten, um in diesen Zustand des „Erlöstseins vom Stress" gelangen zu können, wobei man sich durch die gleichzeitige Leistungssteigerung den folgenden Herausforderungen besser gewachsen fühlte.

\section{Fazit}

Die Vagus-Meditation eröffnet uns neue Chancen gegen Stress, stressbedingte Erkrankungen wie Schlafstörungen, Ängste, Depressionen und Burnout. Sie entspricht unserer körperlichen Anlage und kann somit im Sinne der Salutogenese genutzt werden. Die komplexe Wirkung der Vagus-Meditation steht in Zusammenhang mit dem breiten Leistungsspektrum des 10. Hirnnerven, der nicht nur geflechtartig (Plexusbildung) Herz und Lunge erfasst, sondern auch im Bauchraum einen riesigen Plexus aufweist. Außerdem belegen neue Studien, dass durch Meditation bis ins hohe Alter neue Gehirnzellen gebildet werden können und gleichzeitig die Durchblutung verbessert wird [1]. Somit kann durch Meditation auch ein wichtiger Beitrag gegen gefäßbedingte
Demenz geleistet werden. Weitere Studien belegen einen Anstieg des Glücks- und Leistungshormons Dopamin um 65 \% [2]. Die Vagus-Meditation kann unmittelbar im Stressalltag ihre komplexe Entspannung entfalten, denn viele Menschen tun sich schwer mit der klassischen Meditation in ihrer Grundforderung: „Geh” in die Stille und schließe die Augen". Bei der hohen Dichte zentral zu verarbeitender Sinnesreize wird Stille kaum noch ausgehalten.

\section{Literatur}

1 Lazar SW, Benson H. Function Brain Imaging and Meditation. In: Complementary and Alternative Medicine in Rehabilitation. Leskowitz E (ed), St. Louis: Elsevier Health Sciences, 2002

2 Kjaer TW, Bertelsen C, Piccini P et al. Increased dopamine tone during meditation-induced change of consciousness. Brain Research Cognitive Brain Research 2002; 13: 255259

3 Harvard School of Public Health. Medizinische Praxis Wissenschaft 03/07. In einer Studie an 23681 Griechen zwischen 20 und 86 Jahren konnten Forscher der Universität Athen die Wirkung der meditativen Siesta am Mittag belegen und nachweisen, dass hierdurch das Sterblichkeitsrisiko für HerzKreislauferkrankungen um 37 \% gesenkt werden konnte.

4 Zulley J. Studien der Universität Regensburg ergaben, dass durch 15 Minuten Meditation mittags am Arbeitsplatz die Leistungsfähigkeit am Nachmittag um $35 \%$ anstieg.

5 Schnack G. Der Große Ruhe-Nerv. Freiburg: Kreuz-Verlag, 2012

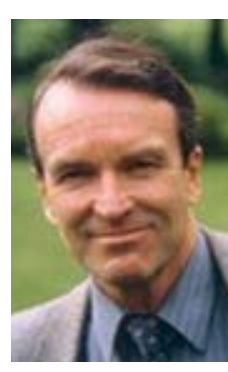

Korrespondenz

Prof. Dr. med. Gerd Schnack Facharzt für Chirurgie/ Unfallchirurgie, Sportmedizin Allensbacher Präventionszentrum Hirschweg 15 78476 Allensbach Tel.: 07533/ 935222 www.praeventionszentrum. com www.vagusmeditation.com 